\title{
Design of Automatic Medication Dispenser
}

\author{
S. Mukund ${ }^{1}$ and N.K.Srinath ${ }^{2}$ \\ ${ }^{1}$ II Year B.E. Student, Department of Electrical Engineering, R.V. College of \\ Engineering, Mysore Road, Bangalore 560059 \\ mukund.srina@gmail.com \\ ${ }^{2}$ Professor and Head of CSE Department, R.V. College of Engineering, Mysore \\ Road, Bangalore 560059, +919845293550 \\ srinathnkervce.edu.in
}

\begin{abstract}
It is necessary to provide medication to the aged in time. Automatic medication dispenser is designed specifically for users who take medications without close professional supervision. It relieves the user of the error-prone tasks of administering wrong medicine at wrong time.

The major components of this medication dispenser are a microcontroller interfaced with an alphanumeric keypad, an LED display, a Motor Controller, an Alarm system, a multiple pill container and dispenser. The overall operation is to facilitate the user to set the timings to dispense multiple pills at required timings. The Alarm system is designed to provide two types of indications - one by lighting an LED and the other by providing a beep sound. The user is required to press a button to get the pill and reset the alarm button. The second alarm is to indicate the optimal availability of the pills in the container to warn the user to refill the dispenser with the required quantity of pills.
\end{abstract}

The major objective is to keep the device simple and cost efficient. The software used is reliable and stable. Elderly population can benefit from this device as it avoids expensive in-home medical care.

\section{KEYWORDS}

Dispenser, microcontroller, motor controller.

\section{INTRODUCTION}

Caring of the aged is of a serious concern in the developing countries. Family members are responsible for the care and management of the old. In the modern age it is difficult for family members to be available all the time to support the aged. Today, in our society most families are nuclear. Elderly would prefer to remain independent and their desire for independence in natural, but it is a worry for their children. Sometimes despite their best effort, the aged fail to remember to take their medication on time. Automatic Medication dispenser is one such approach to help them take their medicines efficiently. As the cost of in-home medical care rises, it has become more and more incumbent among individuals to opt for a device that effectively takes care of their medications. The automatic medicine dispenser serves the purpose.

Natarajan Meghanathan, et al. (Eds): SIPM, FCST, ITCA, WSE, ACSIT, CS \& IT 06, pp. 251-257, 2012. (C) CS \& IT-CSCP 2012 


\subsection{Literature survey}

There are a large variety of medication administration assistance devices for non-professional users. Most of them are manual, providing multiple compartments called pill trays. The pill tray has a number of compartments that can be filled with medication. Each compartment can hold different sizes and combination of medicines. The user is required to take the medicine from each tray each day for a maximum of 28 days. It does not provide any alarm to indicate the time of taking the medicine [1]. Pill-Mate-Medicine reminder is a gadget that uses both visual and audible signals to remind user. It reminds at a pre-set time to take medicines or attend certain events [2]. A smart phone application is designed to help patients to avoid mistakes. It reminds its users to take correct medicines on time and record the in-take schedules for later review by healthcare professionals [3].

\section{METHODOLOGY AND IMPLEMENTATION}

Details about the design of the automatic medicine dispenser (AMD) are included in the paper. Initially the requirements to design this device are collected and then design consideration is taken care. Finally a design process is suggested to design automatic medicine dispenser. The Programmable automatic medicine dispenser designed allows the care taker to reliably administer medications to a patient without needing to be present every time the medication is scheduled. The caretaker pre-programs the AMD that allows it to set up to 21 medications does through an ergonomically designed interface, utilizing an alphanumeric keypad and LCD display. The AMD can be pre-programmed to repeat the same cycle for one month. An alarm is provided to load the medicine if the number of pills/capsules falls below a threshold value that can be fixed by the owner.

\subsection{Customer Requirements}

The major requirements of customers who use this unit are the performance, serviceability, reliability, cost and safety. The performance required are lightweight, ease of use for both the caretaker and the patient, good construction to avoid potential tampering, a Bright warning LED, $70+$ decibel audio alerts and good a display unit.

The product AMD has a long life span and is easy to repair. The software is reliable and mechanical devices are encased for safety and durability. The main features are lightweight, small dimensions, ability to reset the system. A locking mechanism is required in many cases to avoid misusing the unit. Components such as electrical the SMPS and Motors need to be enclosed for safety.

\section{DESIGN CONSIDERATIONS}

Several issues are taken into considerations before starting the design process. In the process of designing the medicine box, issues taken into consideration are: Device has to have a circular base with 30 fans like blades forming compartments. Each compartment should house pills and capsules. Software has to be reliable and enable recording current medication and future medication dispensation. A mechanical lock is to be provided to secure and make it tamper proof. An LED display is to be provided to indicate the working condition and to provide pertinent instructions. Microcontroller interface with LED, Stepper motor, alpha numeric keypad, LCD display and speaker is to be designed. Provision for visual and audio notifications is to be provided. Proper storage is to be ensured for quality of medicine. 


\subsection{Hardware Concept Design}

\section{Power Supply:}

The power supply circuit will provide necessary power requirements for the AMD. Design requirement is $5 \mathrm{VDC}$ for the microcontroller and motor controller. Additionally, $12 \mathrm{~V}$ is necessary for the motor. Current requirements will be dictated mainly by the motor controller design; while the current requirement for the microcontroller is in the range of $100 \mathrm{~mA}$. Also, the power supply may require battery backup to avoid loss of user input selections and time keeping functions.

\section{Keypad and Display:}

The keypad input is a standard 16 key alphanumeric keypad. It enables the user to program the system. The alpha numeric display unit is for the user to view the time set or reset operation. It provides the user visual representation of the contents of the container. The same unit may be used for providing the warning when it is required.

\section{Speaker:}

The Speaker is provided to give a beep sound to warn the patient regarding the time to take the tablet. The same speaker is also use to call the name of the patient which provides the information regarding the updating the pills/capsules into the container. This facility helps the blind to interact with AMD.

\section{Pill Container and Dispenser:}

The pill/capsule container design will have 30 slots with 21 available for medications. The 21 slots will be labelled so that the caregiver can ensure setting the alarm for the correct slot. Additionally, the pill container will incorporate interlock sensors to protect the caregiver and user from harm. Finally the sensors [4] will provide input to the microcontroller for determining access doors status, medication slot positioning and time feedback of the patient accessing the medications.

\section{Microcontroller}

The microcontroller is the main part of the AMD. It is responsible for performing all the functions and commands of the medicine box. The microcontroller is selected to meet the required functionality of the AMD without wasting money on unneeded features. The basic requirements of the microcontroller are a few Input output ports to interface the keyboard, display unit, motor, and speaker. Interrupts are used to enable the user to interact with the system for programming. When the user presses the command key, it enables the user to program the timing and set the required pills to be available in the output poach. The command key also allows the user to program the number of minimum pills/capsules that must be made available before a warning signal is provided. The Microcontroller is so chosen that the system does not require additional external peripheral chips and memory. The memory requirement is $2 \mathrm{~K}$ of READ Only memory and $8 \mathrm{~K}$ of Read writes memory. 


\section{Alarm Module:}

The alarm module will provide an audible alarm tone [9]. This module is designed to send a

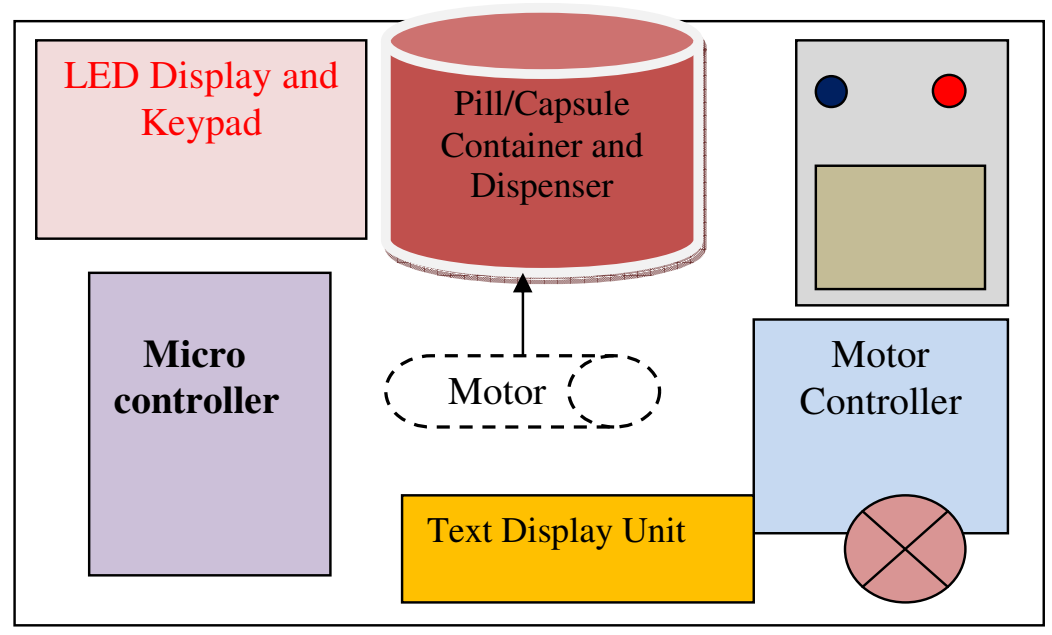

Figure 1 Block Diagram of Medication Dispenser

pattern of audible sounds through the speaker till the patient responds by pressing the button to access the poach for the pills/capsules. An LED is also provided to blink during the alarm process. A visual display string is provided for the user to read the instruction.

A second alarm is provided to prompt the caretaker to load the pills/capsules when the number of pills/capsules in the storage unit falls below the threshold value. The number of pills/capsules threshold value is also programmable. This alarm does not provide audio but gives a warning by blinking.

\section{Motor Controller:}

All motors have a control device called a motor controller to start and stop the motor called a motor controller. It is the actual device that energizes and de-energizes the circuit of the motor so that it can start or stop. The design of the motor controller will be determined by the current requirement of the stepper motor selected. The motor controller takes logic inputs from the microcontroller and supply enough current to the stepper motor to meet maximum torque requirements.

\section{LED:}

The Light emitting diode display will be a simple red light; it provides information such as power on, flashing as he speaker beeps to have the attention of the user and emergency indication. It also provides pre-selected precautions to the patient concerning the medications being currently dispensed. 


\subsection{Complete Design Concept}

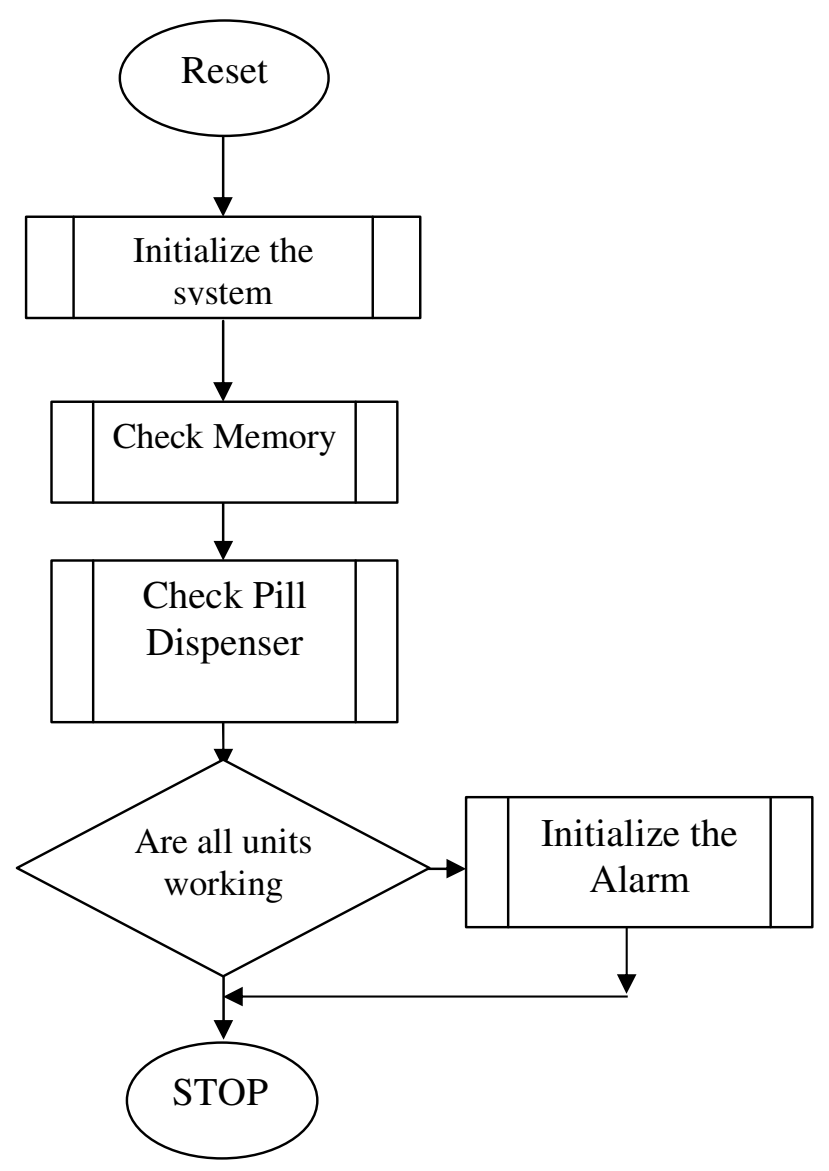

Figure -2 Process when the system in RESET

This includes both the hardware and software concept design. It is shown in Figure -1 block diagram containing the different components of the Medication Dispenser. It contains the Microcontroller which is the heart of the system managing the overall operation of the system. It provides LED display and the Keypad interface to interact with the external world. The text display provides information of the settings and also displays the present setting operation. The Program is written to help the user use the system effectively directing them to use the system without any problem. 


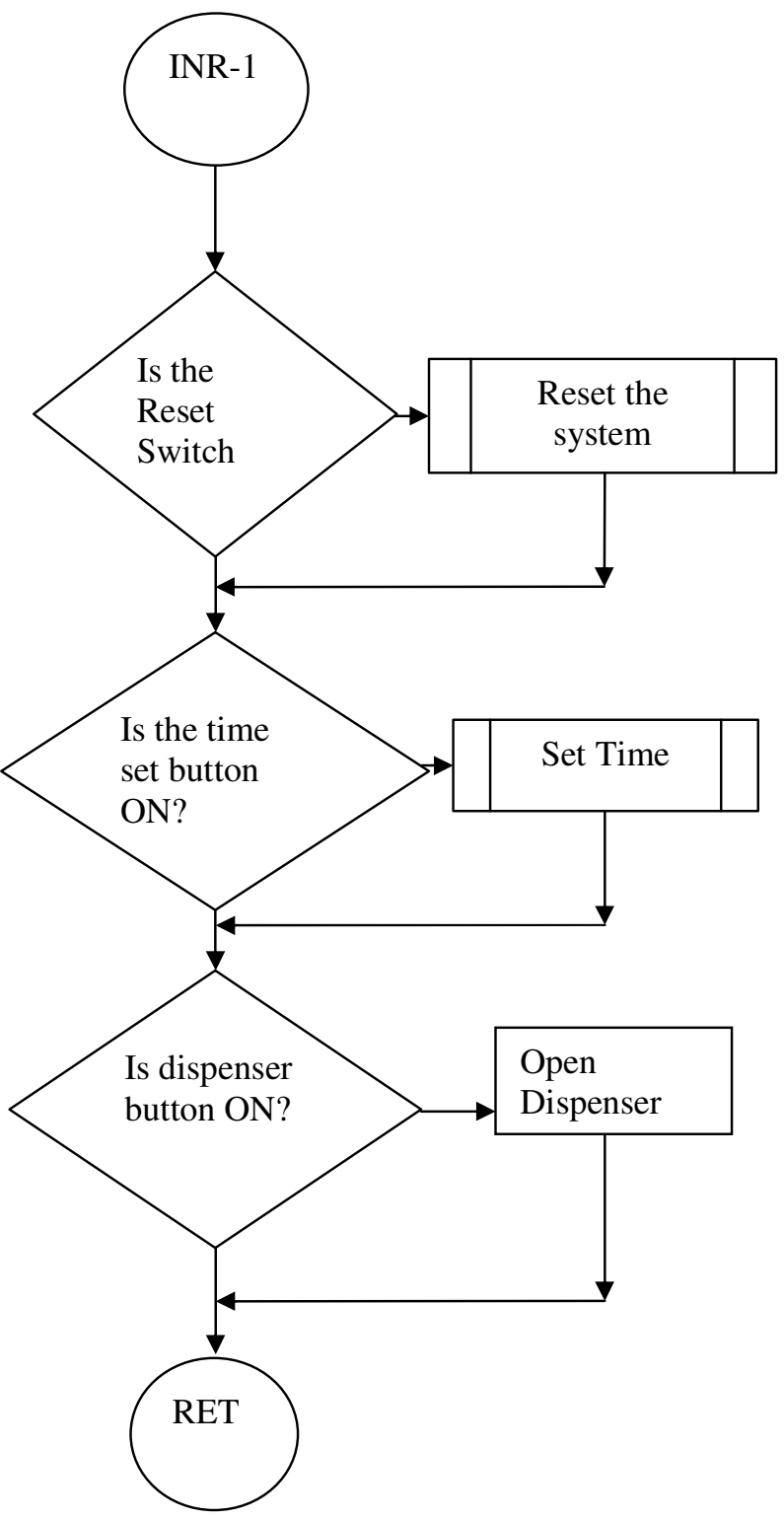

Figure -3 Process when there is Interrupt -1

The Motor controller is programmed so that at the right time the timer internal interrupt interrupts at the right time to rotate and make the pills/capsules drop into the output poach. After the pills/capsules are dropped LED is lit that the user attention can be drawn. It also provides a the Beep sound through the speaker drawing the attention of the patient to take action immediately.

When the user presses the button meant for opening the pouch, the alarm beep sound is switched off, the LED is turned off and the pouch opens for the user to pick the tablets. After the tablet is picked, the user has to press the pouch button so that the pouch closes the door. If the pouch door is not closed within two minutes, the alarm is once again activated. This time, it indicates that the medicine is not picked. It is necessary for the user to pick the pills and close the door. 


\section{Conclusions}

The Automatic Medicine Dispenser is working for pills and capsules of any size. It has been found that the dispenser can be programmed for 31 days for 21 different medicines. It has the facility to send alarms four times a day. It is possible programmable to dynamically change the number of times and the number of pills to be picked as per requirement.

\section{REFERENCES}

[1] "Med-e-lert Medication Pill Box Reminder Dispenser", http://www.amazon.com/Med-E-LertMedication-Pill-Reminder-Dispenser/dp/B0029K03I8

[2] "Alarm Pill Box Pill Mate Event Reminder", http: //www.amazon.com/Alarm-Pill-Mate-EventReminder/dp/B000R3EETI

[3] Mei-Yeing Wang, “A Mobile Phone Based Medicine In-take Reminder and Monitor", 9th IEEE International Conference, June 2009.

[4] Mei-Ying Wang, John K. Zao Wedjat: A Mobile Phone Based Medication Reminder and Monitor.

[5] Kuperman GJ, Bobb A, Payne TH, et al. "MedicationRelated Clinical Decision Support in Computerized Provider Order Entry Systems: A Review" Journal of American Medical Informatics Association, 2007.

[6] Liu JWS, Shih CS, Tsai PH, Yeh HC, Hsiu PC, Yu CY, Chang WH, "End-User Support for Error Free Medication Process," Proceedings of High-Confidence Medication Device Software and Systems, pp. $34-45$,June 2007.

[7] Tsai PH, Shih CS, and Liu JWS, "Algorithms for scheduling multiple interacting medications," Technical Report TR-IIS-08-001, Institute of Information Science Academia Sinica, Taiwan, April 2008.

[8] Tsai, P. H., C. S. Shih, and J. W. S. Liu, "Algorithms for scheduling multiple interacting medications," Institute of Information Science, Academia Sinica, Taiwan, Technical Report TR-IIS08-001, April 2008 Pei Hseun Tseui, "Smart Medication Dispenser: "Design, Architecture and implementation”, IEEE journal, Vol-5, March-2011.

\section{Authors}

Mukund Srinath completed II pre-university examination in the year 2011. He has secured good rank to get merit seat in R.V. College of Engineering, Bangalore in the department of Electrical and Electronics. The major interest is presuming higher students in the Electronics field.

N.K. Srinath is a professor and head of the department of CSE, R.V. College of Engineering. Completed B.E. in engineering from R.V. College of Engineering, M.Tech. from Roorkee University and $\mathrm{PhD}$ from Avinashilingum University for Women.
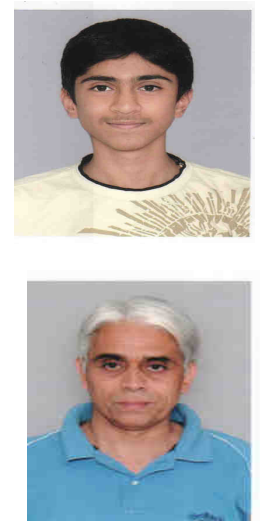